\title{
Regulatory mechanism of ferroptosis, a new mode of cell death
}

\author{
Da Liu, Guoya Li, Ang Jia, Yanyan Zhao, Chenxi Cao, Xuekun Xing* \\ Department of Life Sciences and Technology, Xinxiang Medical University, Xinxiang, Henan 453003, PR China \\ *For correspondence: Email: biyingxiao@163.com; Tel: +86-15637382107
}

Sent for review: 17 April 2018

Revised accepted: 20 October 2018

\begin{abstract}
Ferroptosis is a newly discovered process of cell death that differs from apoptosis, autophagy, and pyroptosis. It is closely related to tumor formation, diseases that damage tissue, and neurodegenerative diseases. Activation of the extracellular regulated protein kinase (EPK) pathway and acylCOA synthetase long-chain family member 4 (ACSL4) are indicative of ferroptosis. During ferroptosis, the mitochondrial volume becomes smaller and the double membrane density increases. The process of ferroptosis involves disruption of the material redox reaction, and changes in the levels of cystine, glutathione, NADPH, and increase of GPX4, NOX, and ROS. Iron increases significantly in ferroptosis. Divalent iron ions can greatly promote lipid oxidation, ROS accumulation, and thus promote ferroptosis. The occurrence and progress of ferroptosis are influenced by multiple factors and signaling pathways.
\end{abstract}

Keywords: Ferroptosis, Iron; Lipid, Active oxygen, Inhibitor, Inducer

\begin{abstract}
This is an Open Access article that uses a funding model which does not charge readers or their institutions for access and distributed under the terms of the Creative Commons Attribution License (http://creativecommons.org/licenses/by/4.0) and the Budapest Open Access Initiative (http://www.budapestopenaccessinitiative.org/read), which permit unrestricted use, distribution, and reproduction in any medium, provided the original work is properly credited.

Tropical Journal of Pharmaceutical Research is indexed by Science Citation Index (SciSearch), Scopus, International Pharmaceutical Abstract, Chemical Abstracts, Embase, Index Copernicus, EBSCO, African Index Medicus, JournalSeek, Journal Citation Reports/Science Edition, Directory of Open Access Journals (DOAJ), African Journal Online, Bioline International, Open-J-Gate and Pharmacy Abstracts
\end{abstract}

\section{INTRODUCTION}

Cell death is a vital process. Cell death modes include programmed death, non-programmed death, and necrosis. In 2012, Dixon et al [1] described ferroptosis as a new mode of nonapoptotic cell death. Observed during study of the mechanism of erastin to kill human fibrosarcoma cells carrying the mutant oncogene RAS, ferroptosis is a mode of non-apoptotic cell death in which cells are injured by iron oxidation [2]. Ferroptosis occurs without typical characteristic of apoptosis, such as caspase activation. Instead, ferroptosis is related to the increase of iron within cells [3]. Ferroptosis is significantly different from autophagy, apoptosis, and pyroptosis both biochemically and morphologically (Table 1) [3]. Recent research has found that ferroptosis is closely associated with many diseases, such as Parkinson's disease (PD) [4], periventricular leukomalacia (PVL) [5], cerebral trauma [6], head and neck cancer (HANC) [7], hepatic carcinoma [8], and esophageal carcinoma [9]. Thus, targeting ferroptosis possibly provides a novel idea for treatment of these diseases.

\section{Characteristics of ferroptosis}

The manifestations of ferroptosis include changes of mitochondria, disruption of redox reaction equilibrium, accumulation of iron, and the build-up of active oxygen. These 
accumulated substances can attack biomacromolecules and cause cell death [3].

The redox reaction of intracellular lipid oxide requires glutathione (GSH) and glutathione peroxidase 4 (GPX4), and the amount of lipid oxide would increase with a decrease in the content of intracellular GSH and decreased GPX4 activity. Instead, $\mathrm{Fe}^{2+}$ oxidizes the lipid in a reaction similar to the Fenton reaction thus producing a large number of reactive oxygen species (ROS) and causing ferroptosis to cells [10]. The long-chain acetyl coenzyme A and the synthetase family 4 (acylCOA synthetase longchain family member 4, ACSL4) are sensitive monitoring indexes of ferroptosis and influencing factors in ferroptosis [11]. In metabolic disorders of intracellular lipid oxide, there can be production of a large number of lipids and lipid metabolites due to the catalytic action of iron ions [3]. At the morphological level, the volume of mitochondria in cells with ferroptosis is decreased and the bilayer of mitochondria is thicker and denser compared to normal cells. Additionally, the crista in the mitochondria decrease or even disappear [1,2]. Inflammatory responses also occur during ferroptosis [12] Overall but these are significantly different from that in cell apoptosis, autophagy, and pyroptosis (Table 1).

\section{Regulatory mechanism of ferroptosis}

The occurrence of ferroptosis is influenced by various factors and regulated by multiple signal pathways. Activation of the extracellular regulated protein kinases (ERK) pathway is considered as an important indicator of ferroptosis, and the extent of ferroptosis is directly proportional to the amount of ERKphosphoric acid [13]. The regulation of cellular ferroptosis is illustrated in Figure 1 and further discussed.

\section{Changes of mitochondria}

In cells undergoing ferroptosis, the mitochondria show significant changes including decreases in volume and bilayer density. Erastin is an antineoplastic drug that can bind to the voltagedependent anion channels (VDACs) of the mitochondrial outer membrane. This can cause VDAC2/3 to close, indirectly inducing the production of ROS, and promoting ferroptosis $[13,14]$. Dixon et al observed that the build-up of

Table 1: Characteristics of ferroptosis, apoptosis, autophagy and pyroptosis

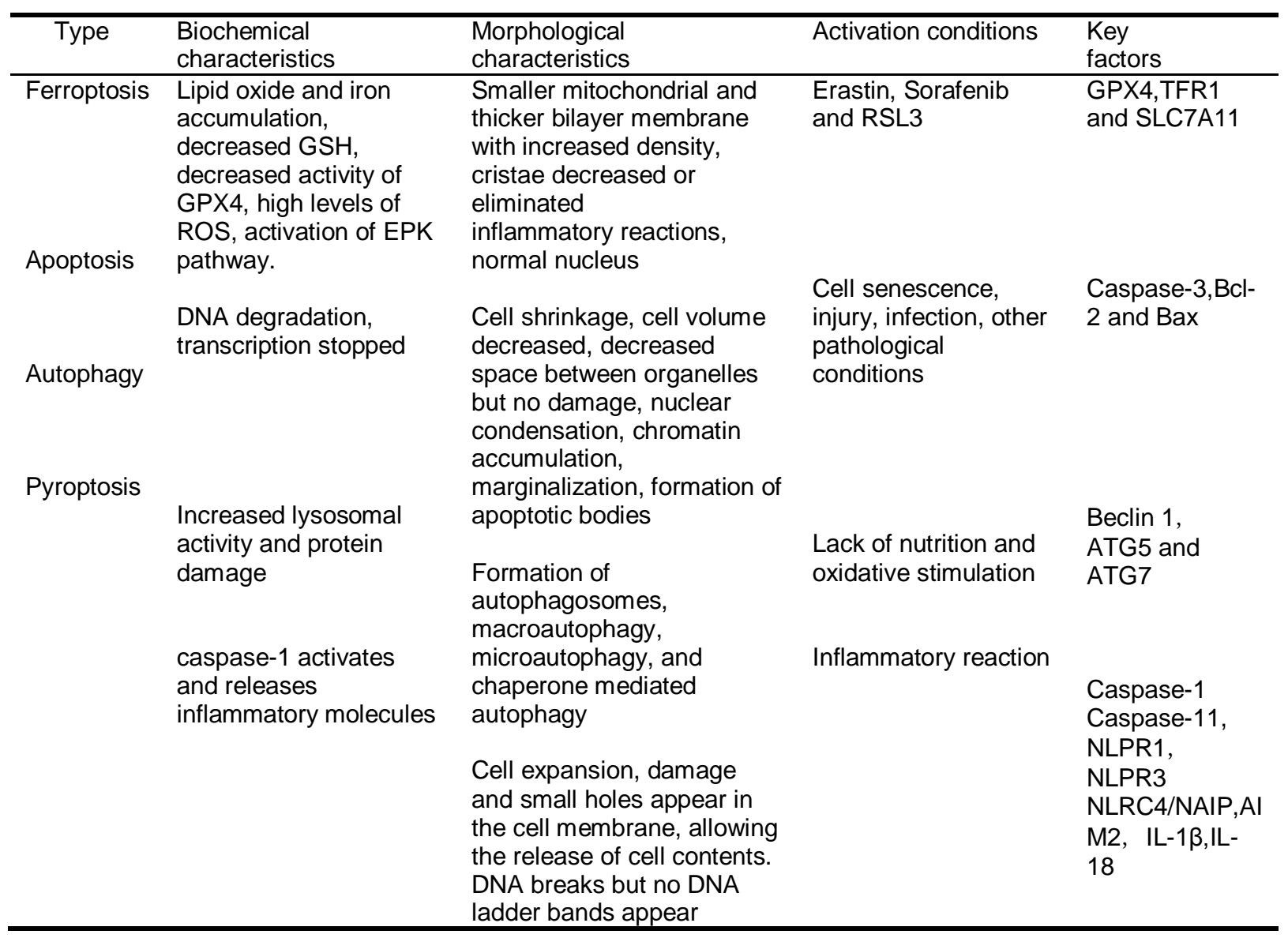




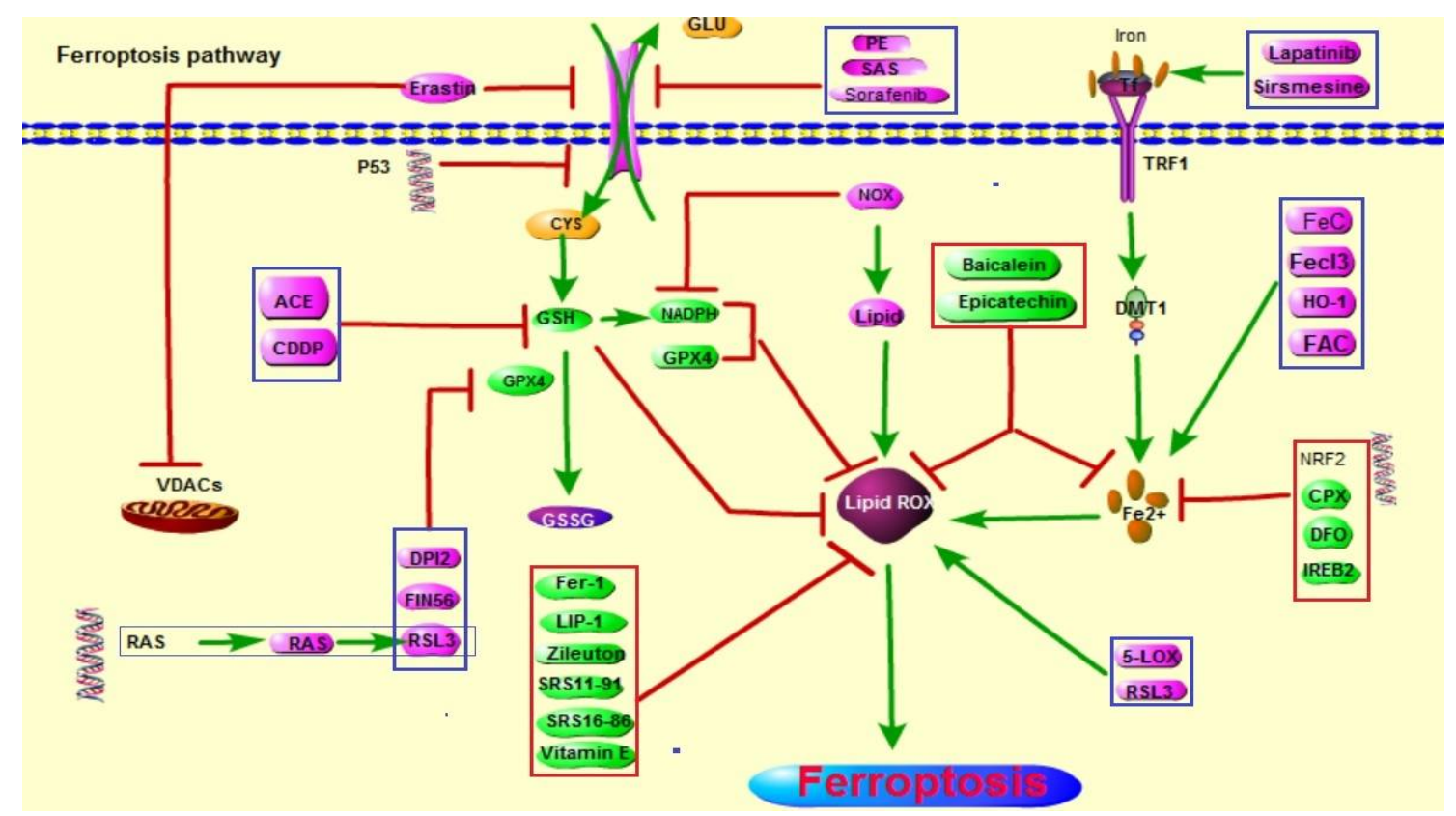

Figure 1: Signal pathway of ferroptosis, a cellular death pathway. Ferroptosis is dependent on iron lipid oxidation damage, which is mainly related to iron metabolism and regulation of intracellular redox reaction. Under some conditions, $\mathrm{XC}$ is inhibited and GSH and NADPH content are decreased, resulting in decreased intracellular antioxidant capacity. In the presence of iron ions, the lipid oxidation process is greatly enhanced, leading to the accumulation of lipid reactive oxygen species. Ultimately, increased lipid reactive oxygen species attack biological macromolecules, causing ferroptosis in cells

active oxygen may lead to mitochondrial damage. In addition, the increase in active oxygen affects calcium ions and the mediation of stress responses of the endoplasmic reticulum [15]. The link of active oxygen changes is critical to study the changes of mitochondria during ferroptosis, the downstream signals of ferroptosis, the regulation pathway of the ferroptosis signal, and the relationship between ferroptosis and endoplasmic reticulum responses. Additionally, recent work provides insight into the mutual metabolism between ferroptosis and other regulatory types of death $[16,17]$.

\section{Regulation of redox reactions}

The process of ferroptosis involves disorders of redox reactions, decreases in the levels of cystine, glutathione, and NADPH, and increases in the levels of GPX4, NOXS, and ROS.

The cystine-glutamate antiporter $(X C)$ on the cell surface is part of an important antioxidation system. The small molecule erastin can initiate ferroptotic cell death by binding and inhibiting VDAC2/3 (described above), and functionally inhibiting the XC cystine-glutamate antiporter system. Thus, cells that are treated with erastin have decreased amounts of cysteine [1]. Because cysteine, glycine, and glutamic acid are required for glutathione synthesis [18], cells treated with erastin are unable to synthesize the antioxidant glutathione. The main role of Glutathione peroxidases (GPX) is to protect against oxidative damage. To do this, glutathione peroxidase acts to reduce lipid hydroperoxides to their corresponding alcohols and to reduce free hydrogen peroxide to water, preventing damage due to oxidation.

With decreased glutathione, there is less antioxidation activity, and the depletion of glutathione leads to excessive lipid peroxidation and cell death. Increased lipid oxidation and ROS promotes ferroptosis under the action of divalent ferrous ions. Related to this pathway, the P53 protein can also inhibit activity of XCsubunit-SLC711, resulting in decreased intracellular cystine and glutathione, increased ROS, and induction of ferroptosis [19].

$\mathrm{NADPH}$ is another important factor affecting the oxidation state. A decrease in the NADPH level promotes the accumulation of ROS and accelerates ferroptosis. Thus, the NADPH level also serves as an indicator of the sensitivity of cells to ferroptosis [20]. NADPH can be 
synthesized via the pentose phosphate pathway, which is inhibited by $\mathrm{H}_{2} \mathrm{O}_{2}$. Intracellular glutathione can act to counter these inhibitory effects of $\mathrm{H}_{2} \mathrm{O}_{2}$. Thus, a decrease in the level of glutathione can result in decreased NADPH level. NADPH and GSSG react to generate GSH, and the occurrence of this reaction is decreased when the content of NADPH decreases [20].

The RAS gene is an oncogene, and expressed RAS induces ferroptosis. RAS can promote the synthesis of RSL3, a class of cell-lethal chemical compounds that promotes cell death. In addition, RAS gene expression promotes the generation of NAPDH oxidase (NOX). NOX acts to decrease the content of NADPH and promote the accumulation of ROS and cell ferroptosis [1]. When the activity of the glutathione oxidase GPX4 is up-regulated, it can inhibit RAS activity. When RAS is inhibited, it is not available to promote RSL3 synthesis, so the RSL3 level decreases, resulting in less cell death. When RSL3 is up-regulated, RSL3 can bind to GPX4 and deactivate it, inhibiting the synthesis of glutamine and promoting the accumulation of ROS [21]. Glutamine is decomposed into glutamic acid, aspartic acid, and alanine, which all participate in the tricarboxylic acid reaction, and are closely associated with carcinogenesis [22]. Glutamic acid also participates in the synthesis of glutathione. In addition, glutamine can be decomposed into L-glutamine [23], which can promote the generation of ROS. Thus, control of glutamine metabolism can achieve ferroptosis occurrence [24].

\section{Regulation of iron metabolic reactions}

Iron has many important functions within the human body, such as the synthesis of hemoglobin, participation in oxygen transport, participation in synthesis of ATP, as the cofactor of some proteins in the electron transport chain of mitochondria, and participation in the synthesis of DNA as the ribonucleotide reductase [25]. The content of iron increases significantly during ferroptosis. Divalent iron ions can significantly promote lipid oxidation, the accumulation of ROS, and ferroptosis.

When ferric ions cannot bind to proteins or other ligands in an appropriate manner, the $\mathrm{H}_{2} \mathrm{O}_{2}-$ dependent Fenton reaction can form ROS with metabolic toxicity. ROS attacks biomacromolecules, thus leading to cell death. Ferritin and its related gene-ferritin light chain (FTL) and ferritin heavy chain 1 (FTH1) can regulate the storage of ferric ions. The heat shock protein B1 (HSPB1) decreases the concentration of intracellular ferric ions by inhibiting the expression of TFR1. Thus, overexpressed HSPB1 can inhibit ferroptosis [26]. Inhibiting the major transcription factor for iron metabolism iron response element binding protein 2 (IREB2) can significantly increase the expression of FTL and FTH1 and inhibit ferroptosis that is induced by erastin [27]. The iron regulator protein (IRP) is a trans-acting factor that can promote the effect of erastin to cause ferroptosis. Heme oxygenase-1 (HO-1) also plays an important role in ferroptosis [3]. The intracellular $\mathrm{HO}-1$ can degrade heme into iron and porphyrin, thus increasing the killing effect of erastin. Overall, the catabolism of hemachrome can promote ferroptosis. The HO-1 inhibitor, zinc protoporphyrin ZnPP, can inhibit ferroptosis induced by erastin. Nuclear receptor coactivator 4 (NCOA4) is a selective carrier receptor for ferroptosis, promoting the degradation of ferritin through autophagy. Overexpressed NCOA4 can increase the degradation of ferritin and promote ferroptosis [28]. Thus, the targeted regulation of ferritin autophagy can regulate iron metabolism in cells, cause iron overloading, and lead to cell injury.

\section{Modulators of ferroptosis}

The development and progression of ferroptosis can be influenced by many factors and multiple signal pathways. Modifiers of ferroptosis will be described in the following paragraphs, including iron element inhibitors, lipid peroxidation inhibitors, iron element inducers, lipid peroxidation inducers, and other modifiers.

\section{Iron inhibitors}

Iron inhibitors include iron chelators such as deferoxamine (DFO), deferoxamine mesylate, 2,2'-bipyridine and others. These iron chelators can effectively inhibit ferroptosis [1]. Additionally, the nuclear factor E2-related factor 2 (NRF2) inhibits ferroptosis by degrading the iron taken in by cells and limiting the production of ROS.

\section{Lipid peroxidating inhibitors}

Lipid peroxidating inhibitors include fertostain-1, liproxstain-1, zileuton, and 5-LOX. Fer-1 is an antioxidant containing arylamine, which can inhibit ferroptosis by inhibiting lipid peroxidation [ 3]. The first and second derivatives of Fer-1 (SRS11-92 and SRS16-86, respectively) exhibit more stable inhibitory activities, resulting in increased tissue damage resistance is strengthened significantly [29]. Liproxstain-1 includes an amide and a sulfamide subunit. Stable and well adsorbed, the drug can significantly inhibit ferroptosis without interfering 
with other cell death modes [30]. The 5lipoxygenase (5-LOX) inhibitor zileuton can inhibit the generation of cellular active oxygen and further inhibit glutamic acid toxicity and cellular ferroptosis, thus exerting neuroprotective effects [31]. The NOX inhibitor dimethylaniline can partially inhibit erastin-induced ferroptosis in cells [1]. Other experiments showed that butylated hydroxytoluene, TROLOX, ebselen, $\alpha$ tocopherol, vitamin E [32], lysosome activity inhibitors such as lostoxin A, pepstatin methyl ester, and ammonium chloride (NH4CL) [33] inhibit ferroptosis by inhibiting lipid peroxidation.

\section{Iron metabolism inducers}

Ferroptosis is related to the increase of iron within cells. Iron metabolism inducers can also induce ferroptosis. Sirsmesine or lapatinib can raise the level of ferric chloride (Fecl3) within the cells and induce ferroptosis by regulating the expression of transferrin in iron metabolism. Sirsmesine and lapatinib can treat breast cancer [34]. The co-incubation with cells of other iron metabolism inducers, such as ammonium ferric citrate, ferric citrate, or ferric chloride can increase the rate of ROS generation and the cell mortality rate [1].

\section{Regulators of oxidative stress}

Erastin, heme oxygenase-1 (HO-1), erastin derivatives such as piperazine erastin (PE) [22] and imidazoline erastin [22] are all regulators of oxidative stress and are likely to induce ferroptosis as they have high solubility and stability in the human body. Sorafenib can induce ferroptosis and increase the toxicity of hepatoma carcinoma cells thus promoting tumor decline [35]. NAPDH oxidase (NOX) acts to produce ROS, and 5-lipoxygenase (5-LOX) also can promote generation of lipid ROS [36] to induce ferroptosis of cells.

\section{Other types of regulators}

Because of the double function of regulating lipid peroxidation and iron metabolism, baicalein has better regulatory effects than deferoxamine ferroptosis inhibitors such as Fer-1 and deferoxamine mesylate, suggesting a good potential for use as therapeutic drugs [37]. Epicatechin regulates lipid ROS metabolism for neuroprotection by regulating nuclear respiratory factors [38] and can also inhibit the ferroptosis pathway for neuroprotection by inhibiting ferroptosis-related genes (Gs, Rpl8, and ATP5G3).
Other regulators such as sulfasalazine (SAS) inhibit cystine adsorption via the cystine/glutamic acid transporters, thus disrupting the redox equilibrium within cells and causing oxidation death dependent on ferric ions [1]. Cisplatin (CCDP) can increase the level of active oxygen within the cells, induce ferroptosis and specific morphological changes and chemical changes in tumor cells, thus inhibiting the growth of tumor cells. Meanwhile, the activity of CDDP can be partially reversed by Fer-1, suggesting that CDDP can induce ferroptosis [39]. As many cancers develop to advanced stages, cancer cells have developed resistance to chemotherapeutics, resulting in the failure of chemotherapy, allowing the tumor to grow and spread further. 1, 2-dioxolane compound (FINO2), a peroxide with five rings that can not only stably and effectively induce ferroptosis of cells in a high-temperature environment but also overcome some common drug resistance. This suggests that it will exert perfect efficacy in cancer treatment [40].

\section{Ferroptosis-related diseases}

\section{Tumors}

Ferroptosis is closely associated with the occurrence, progression, and treatment of many tumors. Sorafenib is often used for the treatment of advanced liver cancer, and the state of the $R b$ protein is an important parameter indicating ferroptosis of liver cancer cells induced by this drug. A decrease in the level of Rb protein may promote ferroptosis in cells [8]. Knock-out of nuclear factor E2-related factor 2 (NRF2) and its signaling pathway downstream target genes can strengthen the activity of sorafenib and inhibit proliferation of liver cancer cells. Acetaminophen can induce ferroptosis in primary hepatic carcinoma cells, and Ferrostatin- 1 can inhibit the induction role of acetaminophen [40]. The NOX inhibitor dimethylaniline partially inhibits the induction of ferroptosis by erastin in human lung cancer. Additionally, glucose-6-phosphate dehydrogenase and phosphoglycerate acid dehydrogenase can also protect human lung cancer cells against erastin-induced ferroptosis when the pentose phosphate pathway is knocked out. Pancreatic cancer cells are highly resistant against apoptosis. Using artesunate for treatment of pancreatic cancer can activate ferroptosis in cancer cells [4]. In breast cancer, downregulation of the $\mathrm{MUCl}-\mathrm{C} / \mathrm{System} \mathrm{Xc}$-signal pathway can induce ferroptosis in triple-negative breast cancer (TNBCA) cells, killing the cancer cells or weakening the cell self-renewal and tumorigenicity [41]. In addition, ferroptosis can occur in renal cancer [8], esophageal carcinoma 
[9], lymphoma [42], head and neck cancer [7], glioma [43], and rhabdomyosarcoma [44].

\section{Tissue damage}

Ferroptosis inhibitor, including iron inhibitor and lipid peroxidating inhibitor can effectively repairs ischemia reperfusion injury (IRI). Tissue damage triggered by ischemia reperfusion, such as myocardial damage, can be treated by inhibiting ferroptosis [24]. The ferroptosis inhibitor SRA1686 can be used to treat renal injury induced by ischemia reperfusion and oxalic acid crystals, rhabdomyolysis, and acute renal failure (ARF) [45]. The ferroptosis inhibitor liproxstain-1 can completely block lipid peroxidation and improve hepatic injury in mice caused by ischemia reperfusion [10].

\section{Neurogenic diseases}

The accumulation of iron in the brain and the generation of ROS promote craniocerebral injury [46]. Hypoxia inducible factor-1 (HIF-1) can regulate the microenvironment under a hypoxia environment through multiple vascular endothelial growth factors and signal pathways. HIF-1 can also reduce the expression of HIF-1 to exacerbate cerebral ischemia. Hypoxia inducible factor-1 prolyl-hydroxylase (HIF PHD) can decompose HIF $[47,48]$. The death of developing oligodendrocytes causes periventricular leukomalacia, and is dependent on iron and is increased by an increase in lipid ROS [49]. In the peripheral nervous system, knockout of GPX4 can cause lipid peroxidation and mitochondrial dysfunction, thus accelerating ferroptosis of neurons and causing paralysis of mice, but only minor effects on cortical neurons of adult rats [12]. The iron chelators, Fer-1, and PKC inhibitor can significantly inhibit the signal pathways of ferroptosis in neurodegenerative diseases [4]. Ferroptosis also plays important roles in various other nervous system diseases such as Huntington's (HD), Alzheimer's disease (AD), motor nerve degeneration, and paralysis [4].

\section{Other diseases}

Wu et al found that ferroptosis participates in the death of keratinocytes due to the loss of GSH. A high dose of vitamin $\mathrm{E}$ can inhibit ferroptosis of skin keratinocytes and reduce skin injury [50]. Mai found that the loss of GPX4 in T cells resulted in accelerated accumulation of lipid peroxide and induction of ferroptosis. Inhibition of ferroptosis can promote the survival and proliferation of $\mathrm{T}$ cells and protect immunological functions [32].

\section{FINAL REMARKS}

Research on ferroptosis is in the preliminary stage and mechanistic details remain to be characterized [1]. The evaluation of ferroptosis primarily is based on morphological changes in cells and the accumulation of ROS. Similar to the activity of caspases in apoptosis, identification of an appropriate marker for ferroptosis will be highly useful [2]. Studies of ferroptosis mechanism have focused on oxidative damage and the iron metabolism signal pathway but additional topics are also worthy of study. For example, many triggers of ferroptosis have been reported in different tissues, but the main signal pathway in ferroptosis remains unclear [3]. Iron regulation and control of oxidation are complex processes that require further analysis [4]. The establishment and optimization of a ferroptosis research model is also an important requirement for more advanced research on ferroptosis [5]. Additionally, it is important to better understand the association of ferroptosis and different diseases to better determine the kind of drugs that might be most effective for disease treatment. Overall, increased study of ferroptosis should facilitate strategies to target this cell death mode as a treatment for many diseases.

\section{DECLARATIONS}

\section{Acknowledgement}

This project was supported by a research grant from the Natural Science Foundation of Henan Province, China (No. 162300410222).

\section{Conflict of interest}

No conflict of interest is associated with this study.

\section{Contribution of authors}

We declare that this work was done by the authors named in this article and all liabilities pertaining to claims relating to the content of this article will be borne by the authors. Da Liu, Guoya Li, Ang Jia, Yanyan Zhao, Chenxi Cao reviewed the literature and wrote the manuscript. Xuekun Xing conceived the idea, reviewed the literature, and edited the manuscript.

\section{REFERENCES}

1. Dixon SJ, Lemberg KM, Lamprecht MR, Skouta $R$, Zaitsev EM, Gleason CE, Patel DN, Bauer AJ, Cantley AM, Yang WS, et al. Ferroptosis: an iron-dependent 
form of nonapoptotic cell death. Cell 2012; 149 (5): 1060-1072.

2. Yang WS, Stockwell BR. Synthetic lethal screening identifies compounds activating iron-dependent, nonapoptotic cell death in oncogenic-RAS-harboring cancer cells. Chem Biol 2008; 15 (3): 234-245.

3. Yang WS, Stockwell BR. Ferroptosis: Death by Lipid Peroxidation. Trends Cell Biol 2016; 26(3): 165-176.

4. Do Van B, Gouel F, Jonneaux A, Timmerman $K$, Gele $P$, Petrault M, Bastide M, Laloux C, Moreau C, Bordet R, et al. Ferroptosis, a newly characterized form of cell death in Parkinson's disease that is regulated by PKC. Neurobiol Dis 2016; 94: 169-178.

5. Bogdan AR, Miyazawa $M$, Hashimoto $K$, Tsuji $Y$. Regulators of Iron Homeostasis: New Players in Metabolism, Cell Death, and Disease. Trends Biochem Sci 2016; 41(3): 274-286.

6. Gascon S, Murenu E, Masserdotti G, Ortega F, Russo GL, Petrik D, Deshpande A, Heinrich C, Karow M, Robertson SP, et al. Identification and Successful Negotiation of a Metabolic Checkpoint in Direct Neuronal Reprogramming. Cell Stem Cell 2016; 18(3): 396-409.

7. Lin R, Zhang Z, Chen L, Zhou Y, Zou P, Feng C, Wang L, Liang $G$. Dihydroartemisinin (DHA) induces ferroptosis and causes cell cycle arrest in head and neck carcinoma cells. Cancer Lett 2016; 381(1): 165-175.

8. Louandre C, Marcq I, Bouhlal H, Lachaier E, Godin C, Saidak Z, Francois C, Chatelain D, Debuysscher V, Barbare JC, et al. The retinoblastoma $(R b)$ protein regulates ferroptosis induced by sorafenib in human hepatocellular carcinoma cells. Cancer Lett 2015; 356(2 Pt B): 971-977.

9. Liu H, Wang X, Shi G, Jiang L, Liu X. Tiam1 siRNA enhanced the sensitivity of sorafenib on esophageal squamous cell carcinoma in vivo. Tumour Biol 2014; 35(8): 8249-8258.

10. Friedmann Angeli JP, Schneider M, Proneth B, Tyurina YY, Tyurin VA, Hammond VJ, Herbach N, Aichler M, Walch $A$, Eggenhofer $E$, et al. Inactivation of the ferroptosis regulator Gpx4 triggers acute renal failure in mice. Nat Cell Biol 2014; 16(12): 1180-1191.

11. Yuan H, Li X, Zhang X, Kang R, Tang D. Identification of ACSL4 as a biomarker and contributor of ferroptosis. Biochem Biophys Res Commun 2016; 478(3): 13381343.

12. Chen L, Hambright WS, Na R, Ran Q. Ablation of the Ferroptosis Inhibitor Glutathione Peroxidase 4 in Neurons Results in Rapid Motor Neuron Degeneration and Paralysis. J Biol Chem 2015; 290(47): 2809728106.

13. Yagoda N, von Rechenberg M, Zaganjor E, Bauer AJ, Yang WS, Fridman DJ, Wolpaw AJ, Smukste I, Peltier $J M$, Boniface JJ, et al. RAS-RAF-MEK-dependent oxidative cell death involving voltage-dependent anion channels. Nature 2007; 447(7146): 864-868.

14. Reed JC, Pellecchia M. Ironing out cell death mechanisms. Cell 2012; 149 (5): 963-965.
15. Dixon SJ, Stockwell BR. The role of iron and reactive oxygen species in cell death. Nat Chem Biol 2014; 10(1): 9-17.

16. Gao $M$, Monian $P$, Pan $Q$, Zhang $W$, Xiang J, Jiang $X$. Ferroptosis is an autophagic cell death process. Cell Res 2016; 26(9): 1021-1032.

17. Sun $X$, Ou Z, Xie $M$, Kang R, Fan $Y$, Niu X, Wang $H$, Cao $L$, Tang $D$. HSPB1 as a novel regulator of ferroptotic cancer cell death. Oncogene 2015; 34(45): 5617-5625.

18. Chen L, Li X, LiU L, Yu B, Xue Y, Liu Y. Erastin sensitizes glioblastoma cells to temozolomide by restraining $x C T$ and cystathionine-gamma-lyase function. Oncol Rep 2015; 33(3): 1465-1474.

19. Jiang L, Kon N, Li T, Wang SJ, Su T, Hibshoosh H, Baer $R$, Gu W. Ferroptosis as a p53-mediated activity during tumour suppression. Nature 2015; 520(7545): 57-62.

20. Shimada K, Hayano M, Pagano NC, Stockwell BR. CellLine Selectivity Improves the Predictive Power of Pharmacogenomic Analyses and Helps Identify NADPH as Biomarker for Ferroptosis Sensitivity. Cell Chem Biol 2016; 23(2): 225-235.

21. Yang WS, SriRamaratnam R, Welsch ME, Shimada $K$, Skouta R, Viswanathan VS, Cheah JH, Clemons PA, Shamji AF, Clish $C B$, et al. Regulation of ferroptotic cancer cell death by GPX4. Cell 2014; 156(1-2): 317331.

22. Chen JQ, Russo J. Dysregulation of glucose transport, glycolysis, TCA cycle and glutaminolysis by oncogenes and tumor suppressors in cancer cells. Biochim Biophys Acta 2012; 1826(2): 370-384.

23. Andrews NC, Schmidt PJ. Iron homeostasis. Annu Rev Physiol 2007; 69: 69-85.

24. Gao M, Monian $P$, Quadri $N$, Ramasamy $R$, Jiang $X$. Glutaminolysis and Transferrin Regulate Ferroptosis. Mol Cell 2015; 59(2): 298-308.

25. Boldt DH. New perspectives on iron: an introduction. Am J Med Sci 1999; 318(4): 207-212.

26. Gao M, Monian $P$, Jiang $X$. Metabolism and iron signaling in ferroptotic cell death. Oncotarget 2015; 6(34): 3514535146.

27. Gammella E, Recalcati S, Rybinska I, Buratti P, Cairo G. Iron-induced damage in cardiomyopathy: oxidativedependent and independent mechanisms. Oxid Med Cell Longev 2015; 2015: 230182.

28. Hou W, Xie $Y$, Song $X$, Sun $X$, Lotze MT, Zeh HJ, 3rd, Kang $R$, Tang $D$. Autophagy promotes ferroptosis by degradation of ferritin. Autophagy 2016; 12(8): 14251428.

29. Zhang Y, Wang H, Li J, Dong L, Xu P, Chen W, Neve RL, Volpe JJ, Rosenberg $P A$. Intracellular zinc release and ERK phosphorylation are required upstream of 12lipoxygenase activation in peroxynitrite toxicity to mature rat oligodendrocytes. J Biol Chem 2006; 281(14): 94609470.

30. Hofmans $S$, Vanden Berghe $T$, Devisscher L, Hassannia $B$, Lyssens $S$, Joossens J, Van Der Veken $P$, Vandenabeele $P$, Augustyns $K$. Novel Ferroptosis

Trop J Pharm Res, November 2018; 17(11): 2315 
Inhibitors with Improved Potency and ADME Properties. J Med Chem 2016; 59(5): 2041-2053.

31. Liu Y, Wang W, Li Y, Xiao Y, Cheng J, Jia J. The 5Lipoxygenase Inhibitor Zileuton Confers Neuroprotection against Glutamate Oxidative Damage by Inhibiting Ferroptosis. Biol Pharm Bull 2015; 38(8): 1234-1239.

32. Matsushita $M$, Freigang $S$, Schneider $C$, Conrad $M$, Bornkamm GW, Kopf M. T cell lipid peroxidation induces ferroptosis and prevents immunity to infection. $J$ Exp Med 2015; 212(4): 555-568.

33. Torii S, Shintoku R, Kubota C, Yaegashi M, Torii R, Sasaki M, Suzuki T, Mori M, Yoshimoto Y, Takeuchi T, et al. An essential role for functional lysosomes in ferroptosis of cancer cells. Biochem $J$ 2016; 473(6): 769-777.

34. Ma S, Henson ES, Chen Y, Gibson SB. Ferroptosis is induced following siramesine and lapatinib treatment of breast cancer cells. Cell Death Dis 2016; 7 e2307.

35. Galmiche A, Chauffert B, Barbare JC. New biological perspectives for the improvement of the efficacy of sorafenib in hepatocellular carcinoma. Cancer Lett 2014; 346(2): 159-162.

36. Yigitkanli K, Pekcec A, Karatas $H$, Pallast S, Mandeville E, Joshi N, Smirnova N, Gazaryan I, Ratan RR, Witztum $J$, et al. Inhibition of 12/15-lipoxygenase as therapeutic strategy to treat stroke. Ann Neurol 2013; 73(1): 129135.

37. Xie $Y$, Song $X$, Sun $X$, Huang J, Zhong $M$, Lotze MT, Zeh $H J R$, Kang R, Tang D. Identification of baicalein as a ferroptosis inhibitor by natural product library screening. Biochem Biophys Res Commun 2016; 473(4): 775-780.

38. Terao J, Piskula $M$, Yao Q. Protective effect of epicatechin, epicatechin gallate, and quercetin on lipid peroxidation in phospholipid bilayers. Arch Biochem Biophys 1994; 308(1): 278-284.

39. Guo J, Xu B, Han Q, Zhou H, Xia Y, Gong C, Dai X, Li Z, Wu G. Ferroptosis: A Novel Anti-Tumor Action for Cisplatin. Cancer Res Treat 2017;

40. Lorincz T, Jemnitz K, Kardon T, Mandl J, Szarka A. Ferroptosis is Involved in Acetaminophen Induced Cell Death. Pathol Oncol Res 2015; 21(4): 1115-1121.

41. Hasegawa M, Takahashi H, Rajabi H, Alam M, Suzuki Y, Yin L, Tagde A, Maeda T, Hiraki M, Sukhatme VP, et al. Functional interactions of the cystine/glutamate antiporter, CD44V and MUC1-C oncoprotein in triplenegative breast cancer cells. Oncotarget 2016; 7(11): 11756-11769.

42. Gout PW, Buckley AR, Simms CR, Bruchovsky N. Sulfasalazine, a potent suppressor of lymphoma growth by inhibition of the $x(c)$-cystine transporter: a new action for an old drug. Leukemia 2001; 15(10): 1633-1640.

43. Ivanov SD, Semenov AL, Kovan'ko EG, Yamshanov VA. Effects of iron ions and iron chelation on the efficiency of experimental radiotherapy of animals with gliomas. Bull Exp Biol Med 2015; 158(6): 800-803.

44. Schott C, Graab U, Cuvelier N, Hahn H, Fulda S. Oncogenic RAS Mutants Confer Resistance of RMS13 Rhabdomyosarcoma Cells to Oxidative Stress-Induced Ferroptotic Cell Death. Front Oncol 2015; 5: 131.

45. Conrad M, Angeli JP, Vandenabeele P, Stockwell BR. Regulated necrosis: disease relevance and therapeutic opportunities. Nat Rev Drug Discov 2016; 15(5): 348366.

46. Wang LF, Yokoyama KK, Chen TY, Hsiao HW, Chiang PC, Hsieh YC, Lo S, Hsu C. Male-Specific Alleviation of Iron-Induced Striatal Injury by Inhibition of Autophagy. PLoS One 2015; 10(7): e0131224.

47. Siddiq A, Aminova LR, Troy CM, Suh K, Messer Z, Semenza GL, Ratan RR. Selective inhibition of hypoxiainducible factor (HIF) prolyl-hydroxylase 1 mediates neuroprotection against normoxic oxidative death via HIF- and CREB-independent pathways. I Neurosci 2009; 29(27): 8828-8838.

48. Siddiq A, Ayoub IA, Chavez JC, Aminova L, Shah S, LaManna JC, Patton SM, Connor JR, Cherny RA, Volitakis I, et al. Hypoxia-inducible factor prolyl 4hydroxylase inhibition. A target for neuroprotection in the central nervous system. J Biol Chem 2005; 280(50): 41732-41743.

49. Larraufie MH, Yang WS, Jiang E, Thomas AG, Slusher $B S$, Stockwell BR. Incorporation of metabolically stable ketones into a small molecule probe to increase potency and water solubility. Bioorg Med Chem Lett 2015; 25(21): 4787-4792.

50. Wu D, Chen L. Ferroptosis: a novel cell death form will be a promising therapy target for diseases. Acta Biochim Biophys Sin (Shanghai) 2015; 47(10): 857-859. 